\title{
Dynamic rupture simulation of the 2011 off the Pacific coast of Tohoku Earthquake: Multi-event generation within dozens of seconds
}

\author{
Hiroyuki Goto $^{1}$, Yojiro Yamamoto ${ }^{2}$, and Saeko Kita ${ }^{3}$ \\ ${ }^{1}$ Kyoto University, Gokasho, Uji, Kyoto 611-0011, Japan \\ ${ }^{2}$ Japan Agency for Marine-Earth Science and Technology, 3173-25 Showa-machi, Kanazawa-ku, Yokohama 236-0001, Japan \\ ${ }^{3}$ Tohoku University, 6-6 Aza-Aoba, Aramaki, Aoba-ku, Sendai 980-8579, Japan
}

(Received December 22, 2011; Revised June 4, 2012; Accepted June 12, 2012; Online published January 28, 2013)

\begin{abstract}
We focus on a complex rupture process, namely, multi-event generation within about 50 seconds during the 2011 off the Pacific coast of Tohoku Earthquake $\left(M_{\mathrm{w}} 9.0\right)$. We perform a $2 \mathrm{D}$ dynamic rupture simulation in order to explain physically the rupture process along the cross-section passing through the hypocenter. Realistic velocity structures are introduced into the simulation model. The dynamic parameters are selected by referring to kinematic source inversion results. The first significant event is generated on the deeper side of the fault. The scattered waves, mainly from the free surface, generate a stick-slip around the hypocenter, and then a second significant event is triggered. The synthetic waveforms consist of two major wave groups that are consistent with the observed ground motions.
\end{abstract}

Key words: The 2011 off the Pacific coast of Tohoku Earthquake, dynamic rupture simulation, strong ground motion.

\section{Introduction}

On March 11, 2011, at 14:46 (JST, GMT+9), the off the Pacific coast of Tohoku Earthquake $\left(M_{\mathrm{w}} 9.0\right)$ hit the eastern part of mainland Japan; the earthquake and, in particular, the great tsunami that followed resulted in the death of more than ten thousand people. Strong ground motions during the earthquake were observed over almost the whole region of Japan by K-NET, KiK-net organized by the National Research Institute for Earth Science and Disaster Prevention (Aoi et al., 2011), and by other seismic networks. At least 18 stations observed over $9.8 \mathrm{~m} / \mathrm{s}^{2}$ of peak ground accelerations in horizontal components, and two stations observed a seismic intensity over 6.5 on the Japan Meteorological Agency scale.

Several research groups have reported the source rupture process during the earthquake. GPS data and low-frequency ground motions imply that a large slip region is located on the shallower side of the seismic fault (Miyazaki et al., 2011; Yoshida et al., 2011). On the other hand, ground motions band-passed within about $0.1-5 \mathrm{~Hz}$ imply that several strong motion generation areas (SMGAs) are located on the deeper side of the seismic fault (Kurahashi and Irikura, 2011; Asano and Iwata, 2012), and the high-frequency radiation area, higher than about $0.5 \mathrm{~Hz}$, is also estimated, by the back-projection method, to be located on the deeper side (Ishii, 2011; Wang and Mori, 2011; Zhang et al., 2011). This indicates that the locations of seismic wave radiation areas depend on the frequency bands (Koper et al., 2011).

Copyright (C) The Society of Geomagnetism and Earth, Planetary and Space Sciences (SGEPSS); The Seismological Society of Japan; The Volcanological Society of Japan; The Geodetic Society of Japan; The Japanese Society for Planetary Sciences; TERRAPUB.
Strong ground motions observed in the Miyagi and Iwate prefectures, close to the epicenter region, consist of two major wave groups with an interval of about $50 \mathrm{~s}$ (e.g. Kurahashi and Irikura, 2011; Nakahara et al., 2011). The origins of the wave groups are estimated to be on the deeper side of the hypocenter, and the origins are located close to each other (Kurahashi and Irikura, 2011; Asano and Iwata, 2012). However, there is a gap of about $50 \mathrm{~s}$ between the rupture times of the corresponding events. The important question is what is the physical mechanism that can explain the time lag. Hereafter, the events are referred to as a "multi-event" in a single earthquake (e.g. Kikuchi and Kanamori, 1982; Sawada et al., 1992).

Duan (2012) has performs 3D spontaneous rupture simulation by considering a subducting seamount just up-dip of the hypocenter. The result shows significant slip near the trench, and the seafloor deformation agrees well with observations (Sato et al., 2011). High-frequency radiations are modeled by three high-strength patches located at the down-dip portion; however, he does not attempt to reproduce details of the high-frequency radiation feature as compared to the actual observed ground motions.

In this study, we carry out a dynamic rupture simulation that models physically consistent slips with stress fields and interface frictions on the fault plane. We especially focus on the numerical modeling of multi-event generation along the dip direction. Ide et al. (2011) have proposed that the major effect can be attributed to a dynamic overshoot due to the fault crossing the free surface, whereas we demonstrate that simulation without the fault crossing the free surface can represent a rupture process consistent with the observed ground motions. 


\section{Method}

Several kinds of numerical methods have been applied to dynamic rupture simulation, and their accuracies have been discussed via a code validation project (e.g. Harris et al., 2009). Most of the methods are categorized as either domain-based methods (e.g., FDM and FEM), or boundarybased methods (e.g., BIEM). Goto et al. (2010) proposed an alternative hybrid approach: viz., the boundary-domain method (BDM). In this method, instantaneous traction change is represented by the combination of a direct term calculated by a boundary-based method for a homogeneous full space, and a residual term calculated from the differences between two solutions for a target heterogeneity model and for a homogeneous full space model by a domain-based method. BDM guarantees accuracy of the stress field close to the fault plane and its applicability to heterogeneous structures.

However, BDM is not applicable to problems with strong heterogeneities beside the fault plane, e.g. faults crossing the material interfaces and faults located on the interfaces of different materials. Actual seismic faults, including the subduction fault corresponding to the earthquake, fall under these conditions. On the other hand, domain-based methods are applicable to model the strong heterogeneities, whereas much denser grids and/or finer meshes than the usual wave propagation problems are required to ensure the accurate and stable simulation of the dynamic ruptures (e.g. Day et al., 2005). Also, special approaches are required to represent the time-dependent dislocations on the fault plane, such as the split-node technique (e.g. Oglesby and Archuleta, 2000; Dalguer and Day, 2007; Kaneko et al., 2011), and it is difficult to embed a low-angle dipping fault with such approaches. This is because a modification of the local coordinate system for FDM (e.g. Kase and Day, 2006) does not simulate well the low-angle case, and a mesh with a high aspect ratio and/or a triangular mesh for FEM give a worse accuracy (e.g. Yoshida, 2010). This means that the domain-based methods for a low-angle dipping fault remain a problem, and they also require great calculation costs to identify an appropriate parameter set for spontaneous rupture simulation.

BIEM for a homogeneous medium has been applied to historical earthquakes to discuss physical properties (e.g. Aochi and Fukuyama, 2002; Fukuyama et al., 2009). The calculation does not exactly follow the actual heterogeneity structures, although some important features have been revealed. Here, we apply BIEM for a homogeneous medium to a spontaneous rupture simulation, and also consider additional terms originating from the heterogeneity by introducing the concept of BDM, namely pseudo-BDM (pBDM). As in the case of BDM, we carry out the following three calculations in parallel: (B) a boundary-based method for a homogeneous full space, (D1) a domain-based method for the target model that we want to simulate, and (D2) a domain-based method for a weak heterogeneity model represented by a layered structure, as shown in Fig. 1. To create the third model, the physical parameters of the fault are homogeneously extended along the normal direction of the fault.

Let $T^{\mathrm{B}}, T^{\mathrm{D} 1}$, and $T^{\mathrm{D} 2}$ be the traction changes calculated

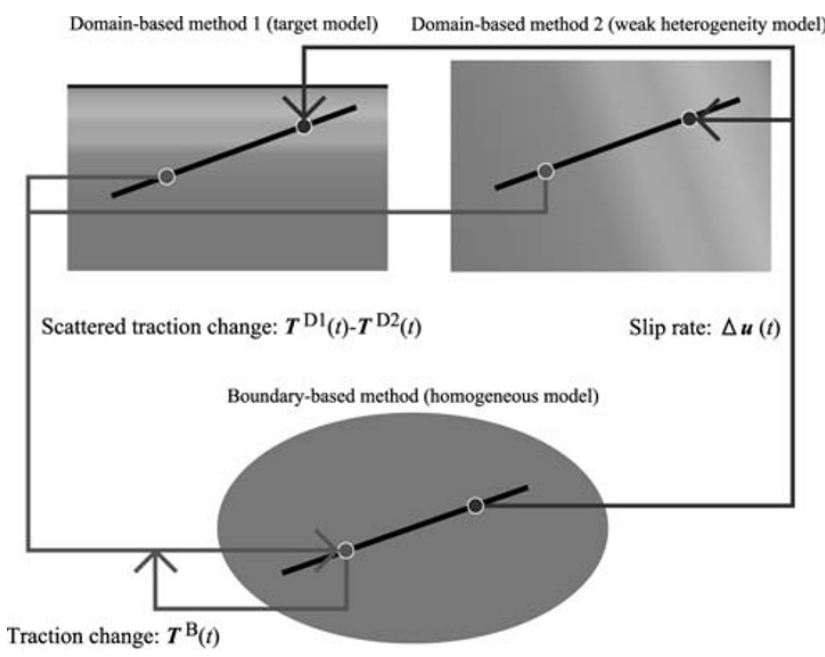

Fig. 1. Calculation procedure of pseudo-BDM (pBDM). pBDM requires three parallel calculations: (1) a boundary-based method for a homogeneous full space (bottom), (2) a domain-based method for the target model (left top), and (3) a domain-based method for a weak heterogeneity model represented by a layered structure (right top). Color gradations in the top figures indicate a conceptual map of material heterogeneity, such as velocity structures, as shown in Fig. 2. An instantaneous traction change is represented by the combination of a direct term calculated from the boundary-based method and a residual term calculated from the differences between two solutions of the domain-based methods.

from the boundary-based method and two domain-based methods, respectively. $T^{\mathrm{D} 1}$ consists of the traction change due to the direct contribution propagated from the slip region, and also the perturbations due to the heterogeneities, e.g. reflected terms from the material interfaces, and refracted terms propagating through the high-velocity zone. On the other hand, $T^{\mathrm{D} 2}$ consists of the terms directly propagated along the fault plane because the fault plane is parallel to the direction of velocity changes. In order to extract the perturbations from $T^{\mathrm{D} 1}$, it is supposed that the difference between $T^{\mathrm{D} 1}$ and $T^{\mathrm{D} 2}$ are the perturbations. Then, we add this to $T^{\mathrm{B}}$ in order to include the effects from heterogeneity. Although the perturbation $T^{\mathrm{D} 1}-T^{\mathrm{D} 2}$ includes the contribution via reflected and refracted waves, the material properties of D2 are not exactly the same as those of B. If the discrepancy between B and D2 is assumed to be small, e.g., the main part of the rupture is located in an almost homogeneous material, the instantaneous traction change $T$ is approximated by the combination of $T^{\mathrm{B}}, T^{\mathrm{D} 1}$, and $T^{\mathrm{D} 2}$, as follows:

$$
T(t) \approx T^{\mathrm{B}}(t)+T^{\mathrm{D} 1}(t)-T^{\mathrm{D} 2}(t) .
$$

The detail scheme in time progress is identical to the BDM introduced by Goto et al. (2010). In the following section, we show some numerical tests such as the representation of the perturbation $T^{\mathrm{D} 1}-T^{\mathrm{D} 2}$, and the small size of spontaneous rupture simulation.

\section{Numerical Model}

We apply pBDM to a 2D $P-S V$ dynamic rupture simulation during the 2011 off the Pacific coast of Tohoku Earthquake, in order to discuss the physical mechanism of multievent generation. The target cross-section, $\mathrm{A}-\mathrm{A}^{\prime}$ in Fig. 2, 

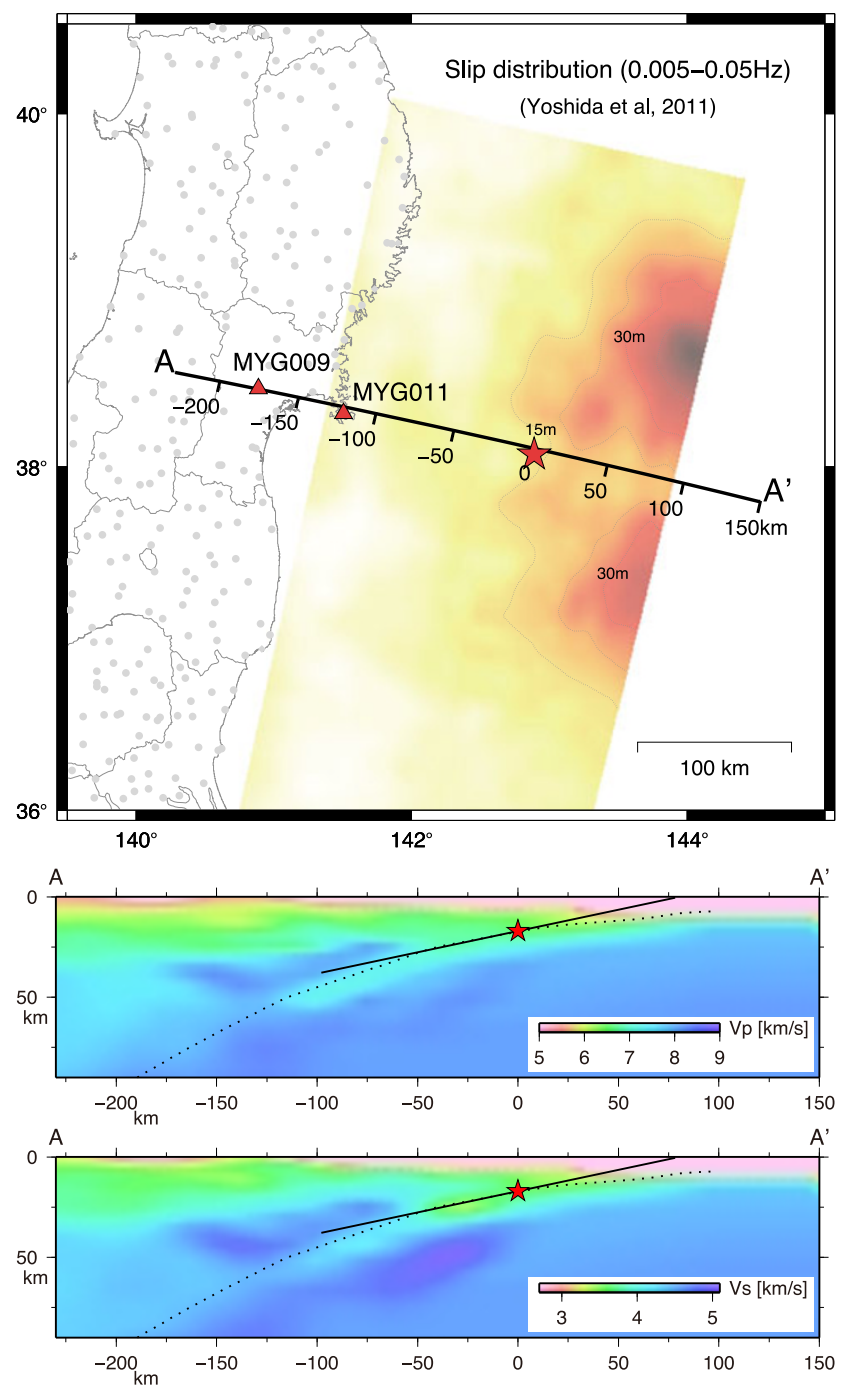

Fig. 2. Geometry of target cross-section (A-A') (top), and velocity models, $V_{p}$ and $V_{s}$, on the cross-section $\left(\mathrm{A}-\mathrm{A}^{\prime}\right)$ (Yamamoto et al., 2011) (bottom). The star denotes the hypocenter of the 2011 off the Pacific coast of Tohoku Earthquake. The circles on the land are the sites recording strong ground motions, and the triangles are the locations of K-NET MYG009 and MYG011. Slip distribution estimated by Yoshida et al. (2011) is plotted together. The thick line on the cross-section is the fault model, and the dotted lines are the depth of the plate boundary (Ito et al., 2005; Miura et al., 2005).

passes through the hypocenter estimated by Yamamoto et al. (2011), and the orientation is normal to the strike direction of the seismic fault $\left(\mathrm{N} 103^{\circ} \mathrm{E}\right)$. The width and depth of the cross-section are $380 \mathrm{~km}$ and $90 \mathrm{~km}$, respectively. Two SMGAs estimated by Asano and Iwata (2012) and Kurahashi and Irikura (2011) are located almost on the cross-section. As shown in Fig. 2, the sites observing strong ground motions are distributed across the mainland. Two of them, K-NET MYG009 and MYG011, are located just on the cross-section.

The tomography model by Yamamoto et al. (2011) is applied to the crust velocity structure on the cross-section. Figure 2 also shows the applied velocity model of a $P$-wave $\left(V_{p}\right)$ and an $S$-wave $\left(V_{s}\right)$. The plate boundary of the North American and Pacific plates (Ito et al., 2005; Miura et al.,
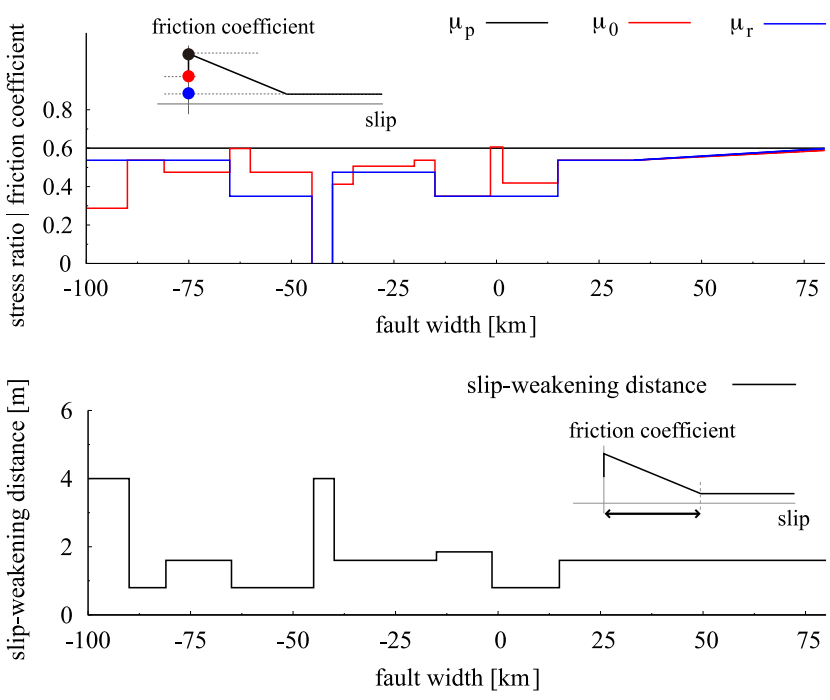

Fig. 3. Dynamic parameter distributions of static and dynamic friction coefficients, and initial stress ratio (top), and slip-weakening distance (bottom).

2005) is also plotted in Fig. 2. We set a planar fault model with a width of $180 \mathrm{~km}$, a dip of $12^{\circ}$, and a hypocenter depth of $17 \mathrm{~km}$, so that the fault model fits the plate boundary around the hypocenter, as shown in Fig. 2. The dip angle and the hypocenter depth coincide with a unified source model by Koketsu et al. (2011). The top of the fault model is located beneath the free surface at a depth of $367 \mathrm{~m}$. This means that the fault edge does not touch the free surface. This assumption may not be realistic, however if the highfrequency radiations can be simulated well without crossing the fault and free surface, the effect of crossing is not always required to simulate the high-frequency radiation. Also, there are no evidences of high-frequency radiations from the top of the fault (e.g. Koper et al., 2011; Asano and Iwata, 2012). If we focus on the high-frequency radiation, the crossing is of not much significance in the simulation.

The boundary integral equation method (BIEM) (Tada and Yamashita, 1997; Goto et al., 2010) is applied as the boundary-based method, and the finite-difference method (FDM) (Levander, 1988) is applied as the domain-based method in pBDM. The velocity models for BIEM are set to be $7.34 \mathrm{~km} / \mathrm{s}$ for $V_{p}$ and $4.20 \mathrm{~km} / \mathrm{s}$ for $V_{s}$, which are the average values on the deeper side of the fault. Density models for both BIEM and FDM are evaluated from the empirical density- $V_{p}$ relation of Ludwig et al. (1970), which has been validated by Miura et al. (2005). The numerical scheme of FDM is of fourth-order accuracy in space, and of secondorder accuracy in time using staggered grids. The element size of BIEM is $87.9 \mathrm{~m}$, and the grid intervals of FDM are homogeneously $100 \mathrm{~m}$. The time step intervals of both methods are set at $4.77 \times 10^{-3} \mathrm{~s}$. The Courant-FriedrichsLewy conditions for BIEM and FDM are 0.399 and 0.40, respectively. The maximum support frequency of FDM is $1.34 \mathrm{~Hz}$. Internal damping of the crust structure is set to be $Q=100 f$ and installed by using the approach of Graves (1996).

Friction on the fault plane obeys a linear slip-weakening 

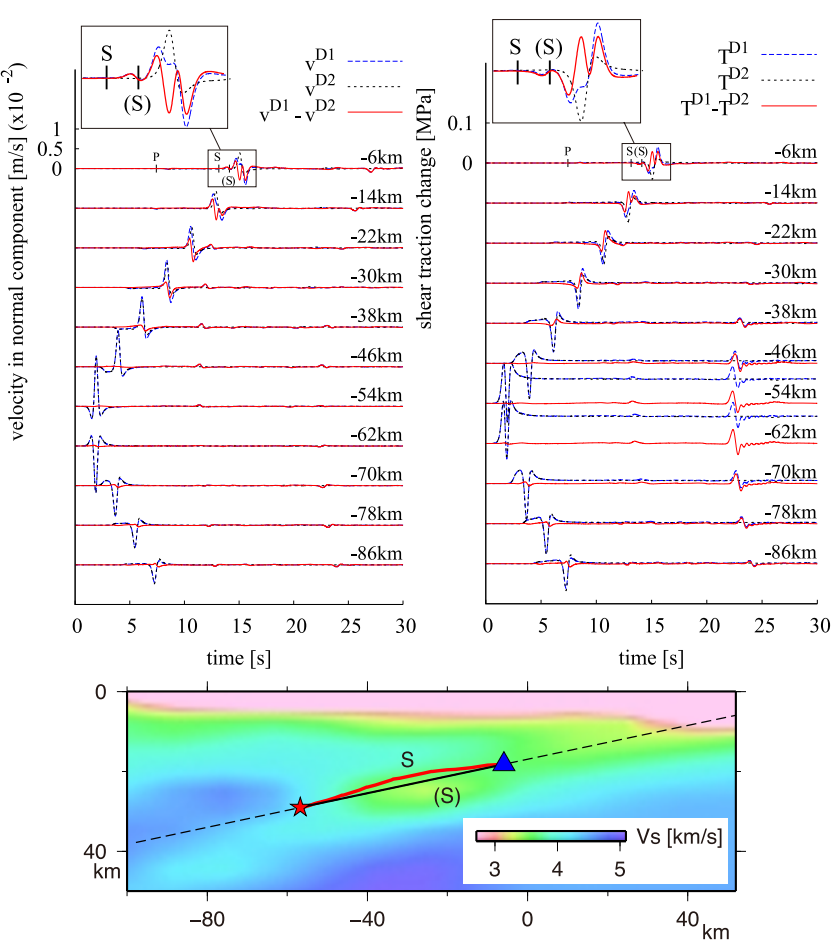

Fig. 4. Characteristics of perturbation terms calculated from the differences between solutions from the target model (D1) and the layered model (D2); velocities in normal component and shear traction changes observed along the fault plane (top). A double-couple point source is located at $-58 \mathrm{~km}$ along the fault width. Seismic ray traces of $S$-wave for $\mathrm{D} 1, S$, and for $\mathrm{D} 2,(S)$ (bottom).

friction law (Ida, 1972), and the rate-dependency of friction is not considered. In such a case, the dynamic parameters consist of the initial stress ratio $\mu_{0}$, static and dynamic friction coefficients $\mu_{p}, \mu_{r}$, and the slip-weakening distance $D_{C}$. The stress ratio is defined by dividing the initial shear traction by the normal stress. When the shear traction is unloaded, the value of the last friction strength is stored. After the traction reaches the friction strength again, the slip is allowed. This assumption is consistent with a standard elasto-plastic constitutive model.

Figure 3 shows the distributions of the dynamic parameters, which are evaluated by trial and error in order to represent the location of SMGAs by Asano and Iwata (2012), while a priori information about the plate couplings and the inflections is not considered. The origin of the axis along the fault coincides with the hypocenter. A constant value of static friction, 0.6, is assumed over the fault. A 3-km-wide nucleation zone is located around the hypocenter from -1.5 to $1.5 \mathrm{~km}$, where the initial stress ratio, $\mu_{0}=0.60625$, slightly exceeds the static friction coefficient, $\mu_{p}=0.6$. The stress condition allows the rupture to start from the nucleation zone (e.g. Fukuyama and Madariaga, 1998). Two high stress drops are assumed in the deeper region: (1) from -65 to $-45 \mathrm{~km}$, and (2) from -35 to $-15 \mathrm{~km}$. The former region assumes $\mu_{r}=0.35, \mu_{0}=0.475 \sim 0.598$, and $D_{C}=0.8 \mathrm{~m}$. The latter region assumes $\mu_{r}=0.475$, $\mu_{0}=0.506 \sim 0.538$, and $D_{C}=1.6 \mathrm{~m}$. The barrier region, with a $5-\mathrm{km}$ width located from -45 to $-40 \mathrm{~km}$, does not
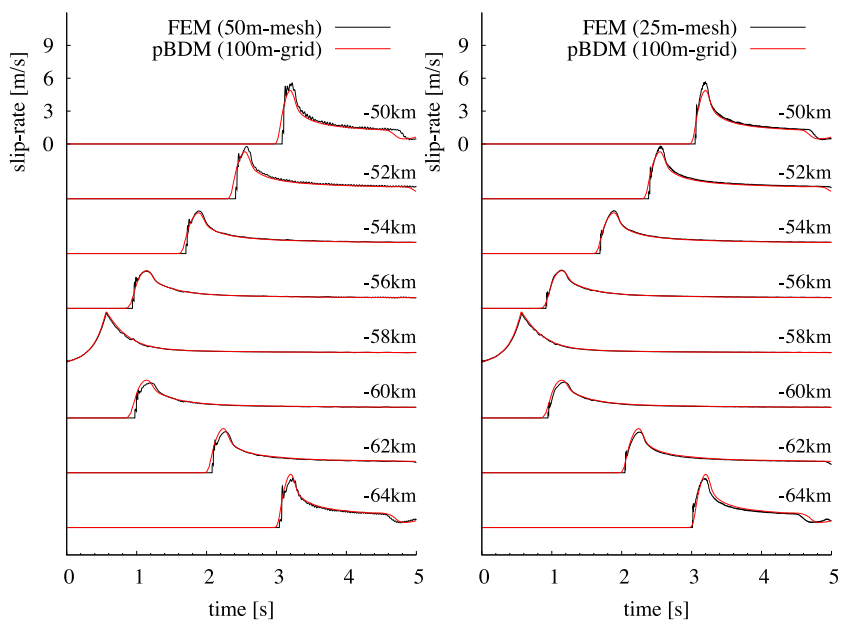

Fig. 5. Comparison of slip-rate time histories calculated by pBDM and FEM. The mesh size of FEM is set to be $50 \mathrm{~m}$ (left), and $25 \mathrm{~m}$ (right).

allow slip. Regions with negative stress drops and a large slip-weakening distance are located at the bottom of the fault in order to represent a ductile rupture and a yielding of the surrounding materials (Aochi and Fukuyama, 2002). The dynamic friction increases in the shallower region in order to reduce the slip-rate amplitudes (Wada and Goto, 2012), because the stopping front from the shallower edge is assumed to be less dominant in this simulation. This assumption is consistent with the hypothesis that the effect of crossing the fault to free surface is not significant in generating high-frequency radiation. Uniform distribution of the normal stress, $160 \mathrm{MPa}$, is assumed, and normal stress changes due to the rupture are considered in this simulation.

In order to check the performance of $\mathrm{pBDM}$ to the velocity model, we show the results of two simple tests; (1) to see the perturbation waves under the excitation from a double-couple point source, and (2) to see the rupture propagations. First, we examined the point source problem. The double-couple source is located at $-58 \mathrm{~km}$ along the fault width, the seismic moment per unit length is $4.74 \mathrm{GN}$, the arms of the moment correspond to the fault dip angle, and smoothed ramp function with $1.0 \mathrm{~Hz}$ of the center frequency is adopted to the source time function. Figure 4 shows the calculated velocities in the normal component to the fault, and the calculated shear traction changes on the fault plane. $v^{\mathrm{D} 1}$ and $T^{\mathrm{D} 1}$ are calculated from the model D1, the target model, and $v^{\mathrm{D} 2}$ and $T^{\mathrm{D} 2}$ are from $\mathrm{D} 2$, the layered model. The perturbations $v^{\mathrm{D} 1}-v^{\mathrm{D} 2}$ and $T^{\mathrm{D} 1}-T^{\mathrm{D} 2}$ around the point source from -70 to $-46 \mathrm{~km}$ are smaller than $v^{\mathrm{D} 1}$ and $T^{\mathrm{D} 1}$ themselves. For $T^{\mathrm{D} 1}$, additional phases are observed around $20-25 \mathrm{~s}$, and $T^{\mathrm{D} 1}-T^{\mathrm{D} 2}$ extracts the phases considered to be the effect from the heterogeneity. From -22 to $-6 \mathrm{~km}$, the arrival time of the main phase of $v^{\mathrm{D} 1}$ is faster than $v^{\mathrm{D} 2}$, and causes the amplitude of perturbations to increase. Since refracted waves for model D2 are not observed along the fault plane, the faster phase corresponds to the refracted waves considered to be the contribution from the velocity heterogeneity. Figure 4 also shows the seismic ray traces of the $S$-wave, $S$ and $(S)$, corresponding to model D1 and D2, re- 

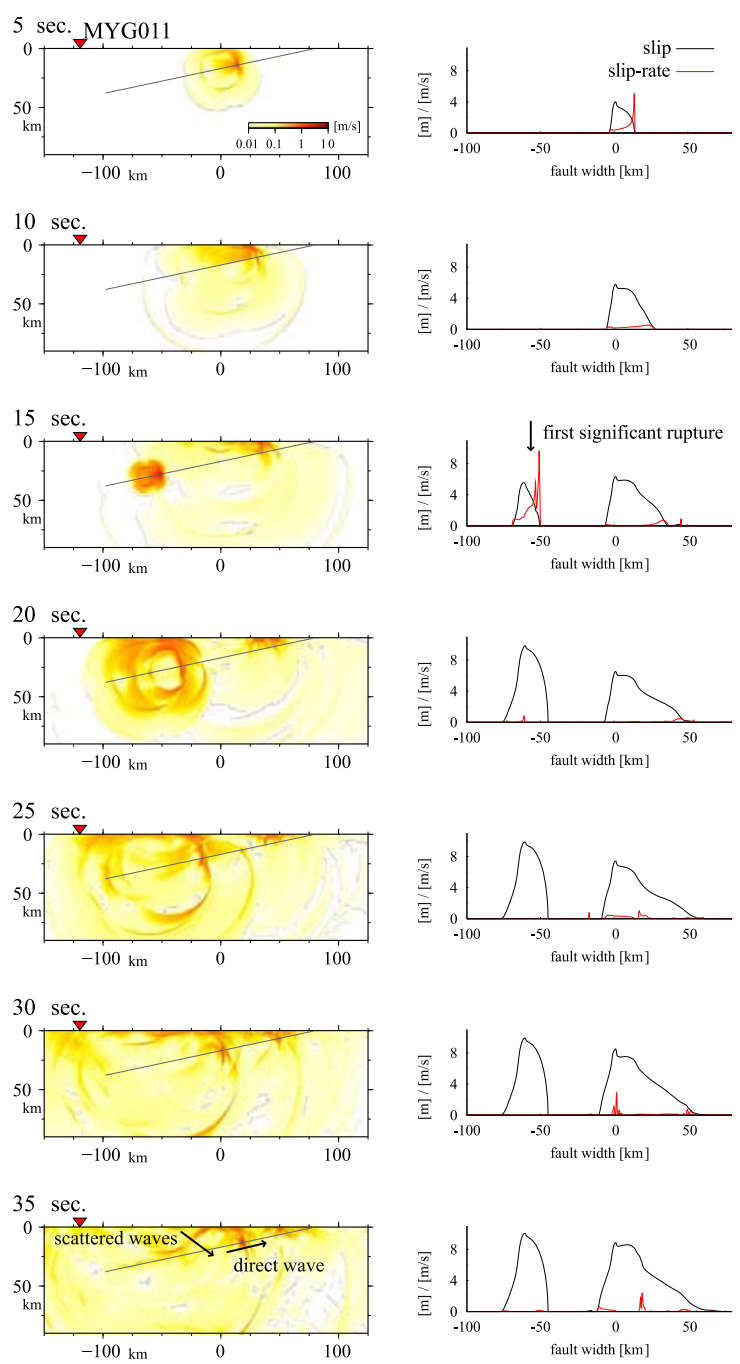
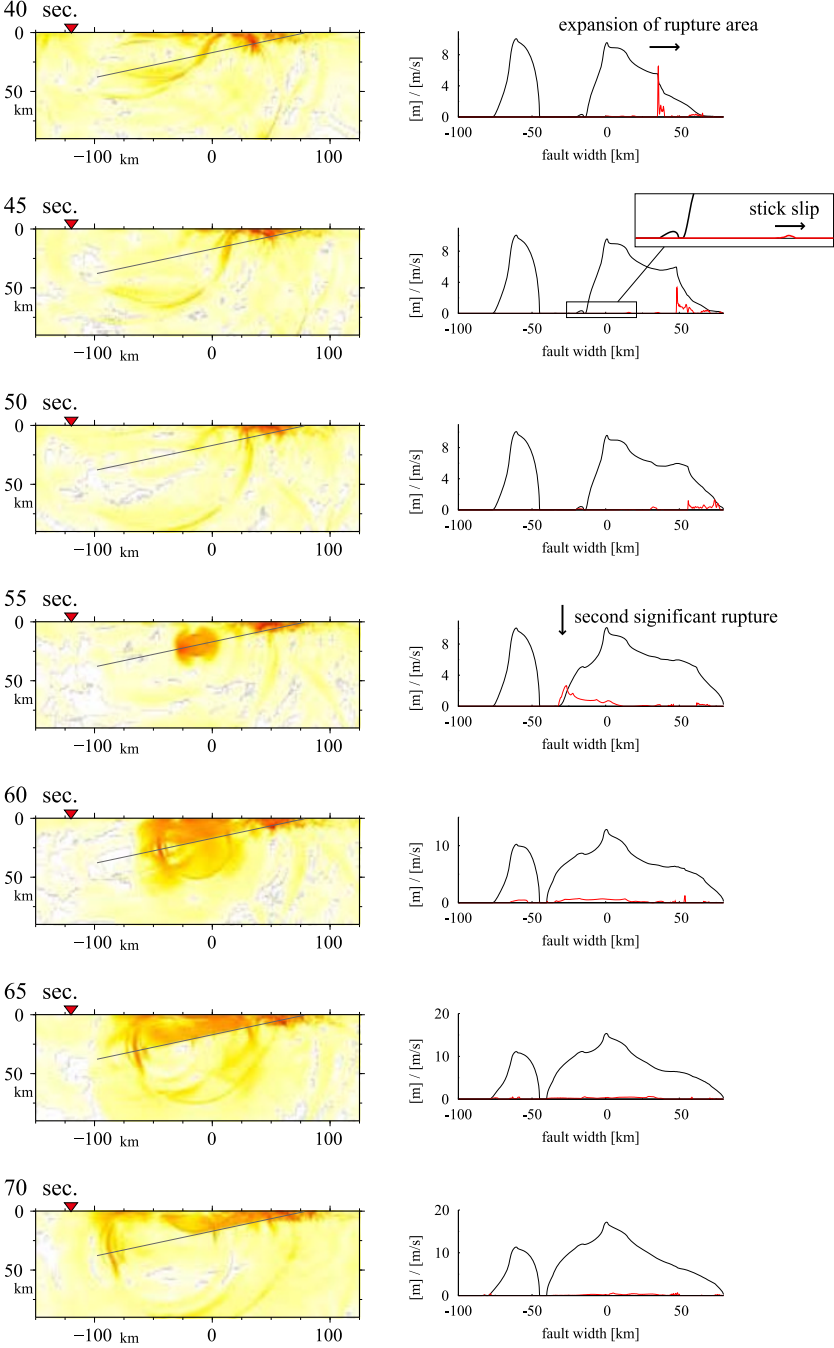

Fig. 6. Snap shots of velocity wave fields and slip and slip-rate distributions on the fault plane. At each time step, the color maps show the absolute values of particle velocity (left column). The black and red lines show the distribution of slip and slip-rate, respectively (right column).

spectively, and travel times, $13.15 \mathrm{~s}$ and $14.12 \mathrm{~s}$ at $-6 \mathrm{~km}$, are also marked on the velocity and traction change. The differences of ray traces and travel times indicate that the perturbations contain the refraction waves, and the travel times of the perturbations are well calculated. Therefore, we assume that we are able to discuss the time gaps due to the perturbations based on the results of pBDM.

We also perform another rupture simulation for a trimmed area of $30 \times 50 \mathrm{~km}^{2}$ from the velocity model. We focus on the area around the first high stress drop region. A fault model with a width of $24 \mathrm{~km}$ trimmed from the original model is adopted, and a $3-\mathrm{km}$-wide nucleation zone is set to be around $-58 \mathrm{~km}$ along the fault width. The dynamic parameters are set to be $\mu_{p}=0.6, \mu_{r}=0.35$ and $D_{C}=0.8 \mathrm{~m}$ over the whole fault plane, and $\mu_{0}=0.60625$ in the nucleation zone and $\mu_{0}=0.41875$ in the other region. The velocity and density models are the same as with the simulation model. For the reference solutions, a finiteelement method (FEM) with split nodes on the fault plane is applied (e.g. Oglesby and Archuleta, 2000). Two different sizes of mesh layout are adapted to the fault, and the mesh sizes are, on average, $50 \mathrm{~m}$ and $25 \mathrm{~m}$, respectively. Figure 5 shows the slip-rate time histories calculated by pBDM and
FEM. The synthetic slip-rates from pBDM agrees well with the FEM results, even though pBDM uses coarser grid intervals than the size of the FEM mesh. This supports the applicability of pBDM, at least in the deeper side of the fault model.

\section{Results and Discussions}

Figure 6 shows snapshots of the absolute values of the simulated particle velocities, and slip and slip-rate distributions, on the fault plane, and Fig. 7 shows a comparison of initial and final shear tractions. Rupture is initiated at the hypocenter within $10 \mathrm{~s}$. Seismic waves propagate in the deeper and shallower directions of the fault width, and then the first significant event is triggered at around $-60 \mathrm{~km}$ after about $15 \mathrm{~s}$. The event generates seismic waves of large amplitude in the shallower direction. The seismic waves consist of a direct wave propagating along the fault plane and scattered waves mainly reflecting on the free surface. When the direct waves reach the vicinity of the hypocenter where slips have occurred in the initial stage, the slips are excited again within 25-35 s. The direct waves also expand the rupture area to the shallower region in about 35-55 s. Ide et al. (2011) have suggested that the first significant rupture oc- 


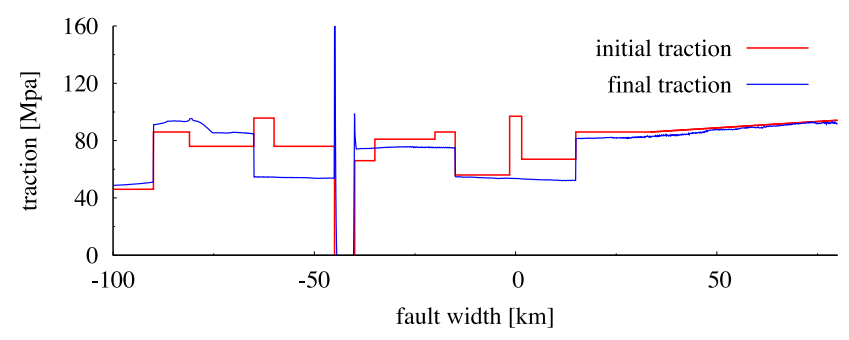

Fig. 7. Comparison of initial and final shear tractions. The differences indicate the stress drop of the simulation.

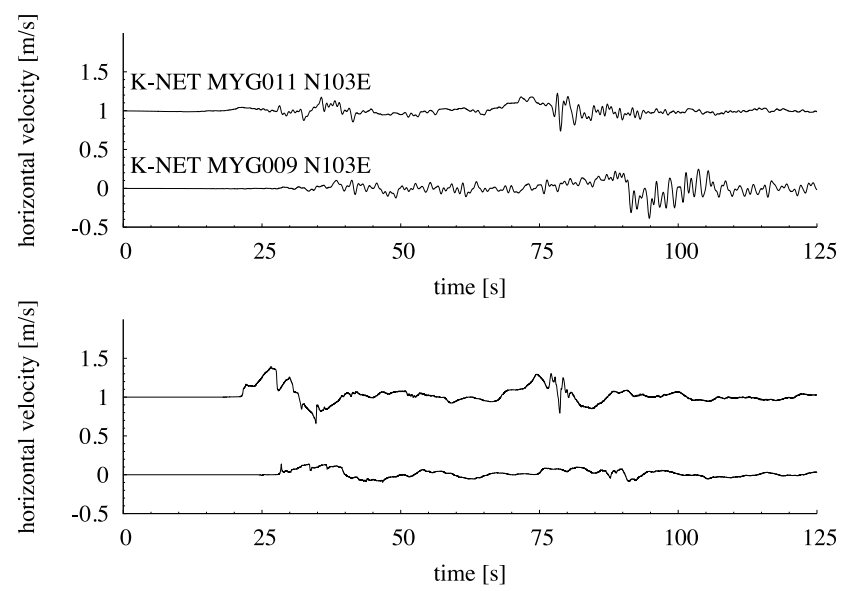

Fig. 8. Observed velocity waveforms at K-NET MYG011 and MYG009 (top), and the corresponding synthetic velocity waveforms (bottom).

curred on the deeper side, and that the rupture propagated toward the shallower side. The simulated rupture process is consistent with Ide et al. (2011).

At the same time, the scattered waves reach the fault plane after $40 \mathrm{~s}$, and generate a stick-slip with low sliprates around the hypocenter. Here, the stick-slip means that slips in the region repeat the stopping and rupturing. This causes a crack growth gradually expanding the ruptured area in the deeper direction, and then the second significant event is triggered at around $-30 \mathrm{~km}$ after about $55 \mathrm{~s}$. This event generates seismic waves of large amplitude propagating mainly in the landward direction.

Synthetic velocity waveforms at the location corresponding to K-NET MYG011 and MYG009 (see Fig. 2) are compared to the observed velocity waveforms at MYG011 and MYG009, as shown in Fig. 8. The observed waveforms are $\mathrm{N} 103^{\circ} \mathrm{E}$ components parallel to the cross-section and band-passed within $0.05-1.35 \mathrm{~Hz}$. Notice that the synthetic waveforms are calculated for a $2 \mathrm{D} P-S V$ wave field, and the amplitudes and the phases should not be directly compared. The synthetic waveforms show two significant phases with an interval of about $50 \mathrm{~s}$, which is consistent with the observed waveforms. The first phase, at about 20-50 s, is generated from the first event, which took place at about $-60 \mathrm{~km}$ along the fault at about $15 \mathrm{~s}$. The second phase, at about $65-100 \mathrm{~s}$, is generated from the second event, which took place at about $-30 \mathrm{~km}$ along the fault at about $55 \mathrm{~s}$. The second phase of both the observed and synthetic waves contains an initial low-frequency stage, at about 65-75 s for MYG011, and a following high-frequency phase. The

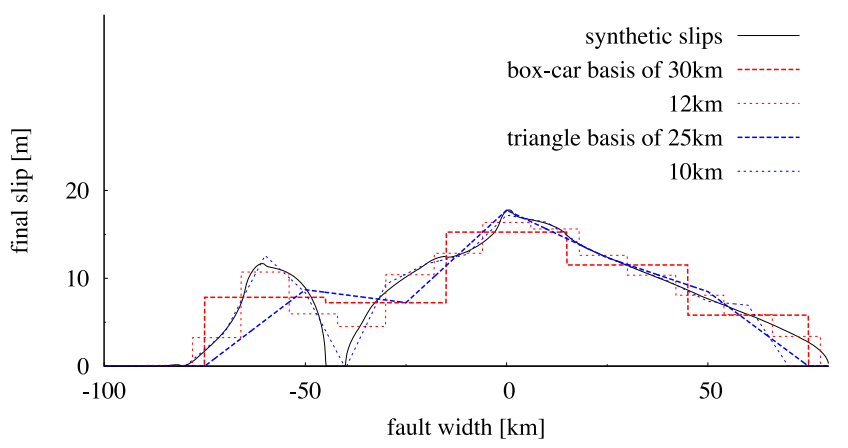

Fig. 9. Best fit models of the simulated slip distribution with a superposition of spatial basis functions, (1) box-car basis and (2) triangle basis.

time intervals between the first and second phases correspond to the interval between the ruptures at about $15 \mathrm{~s}$ and $55 \mathrm{~s}$. Within these $40 \mathrm{~s}$, the first $25 \mathrm{~s}$ corresponds to the time interval of the scattered waves, and the following $15 \mathrm{~s}$ corresponds to the time for crack growth.

The barrier set in the deeper side of the fault may play an important role in the generation of the multi-event. In contrast, no clear barriers are seen in the rupture processes estimated by kinematic source inversions. We suppose that the barrier of our model is consistent with the rupture processes because the spatial resolutions in the inversions may not be sufficient to reveal it, this has been discussed in the Source Inversion Validation project (Page et al., 2011). For the earthquake, we select four inversion results by Ide et al. (2011), Miyazaki et al. (2011), Suzuki et al. (2011), and Yoshida et al. (2011), to compare the maximum spatial resolutions in their inversion schemes. The slip distributions are represented by the superposition of the basis functions such as the box-car basis (Suzuki et al., 2011; Yoshida et al., 2011) and the triangle basis (Ide et al., 2011; Miyazaki et al., 2011). The spatial sizes, e.g. the size of sub-faults (Suzuki et al., 2011; Yoshida et al., 2011), are usually selected such that the dominant frequency caused by the spatial periodicity is well outside the frequency range of analysis (e.g. Sekiguchi et al., 2000). In addition, the coefficients for each basis are constrained by prior information in the Bayesian approach such as Akaike's Bayesian Information Criterion (ABIC) (e.g. Ide and Takeo, 1997; Sekiguchi et al., 2000), and the constraint results in the slip distribution being smoothed.

We consider an ideal condition to estimate the slip distribution from the slip data obtained just on the fault plane without any observation noises. Figure 9 shows best fit models of the simulated slip distribution with a superposition of spatial basis functions. The spatial sizes of each basis correspond to the parameters of Suzuki et al. (2011), Yoshida et al. (2011), Miyazaki et al. (2011) and Ide et al. (2011), respectively. Three of the models cannot estimate the existence of the barrier. One model represented by a triangle basis of $10 \mathrm{~km}$ intervals detects no slip region at $-40 \mathrm{~km}$. The model would be obtained if the records obtained only on the fault plane were available. However, in the actual situation, smoothing is necessary because of constraints.

Kurahashi and Irikura (2011) and Asano and Iwata 

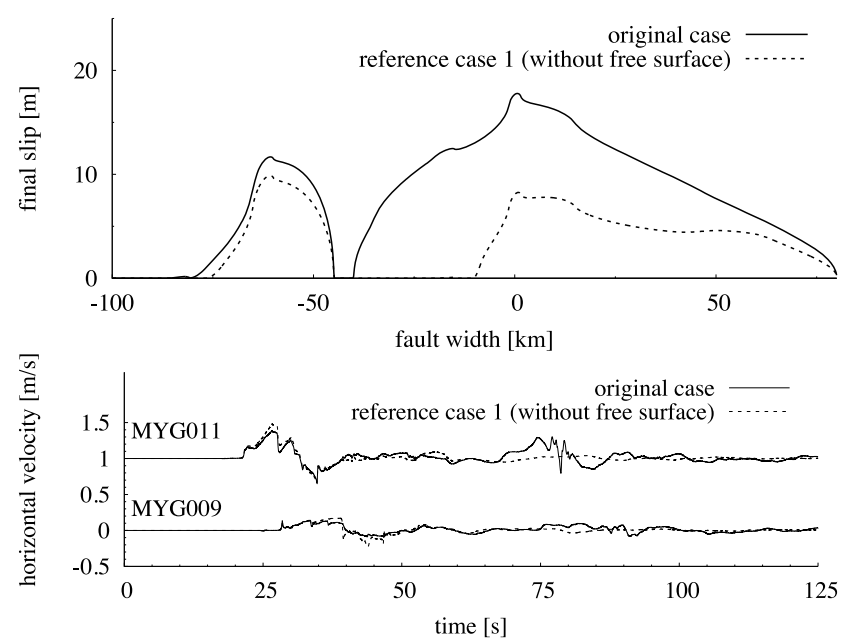

Fig. 10. Comparison of simulation results between the original case and reference case 1 (without free surface); final slip distribution (top) and synthetic velocity waveforms (bottom).
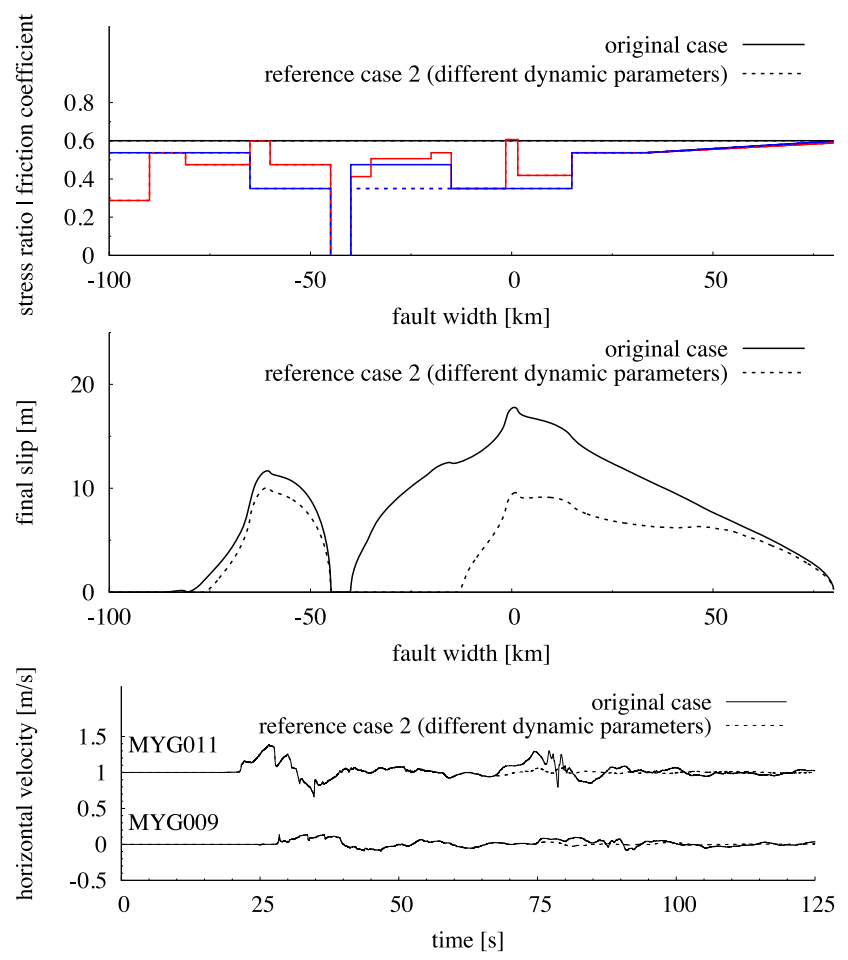

Fig. 11. Comparison of simulation results between the original case and the reference case 2 (different dynamic parameters); friction coefficient (top), final slip distribution (middle) and synthetic velocity waveforms (bottom).

(2012), have estimated the locations of SMGAs. Constant stress drops and square areas of SMGAs are assumed, and the locations, rise time, rupture starting point, and the stress drop are estimated by a trial-and-error approach (Kurahashi and Irikura, 2011), and a grid search (Asano and Iwata, 2012). The procedures infer that a spatial variation within the size of SMGA is averaged. In addition, the relative locations of the two SMGAs are opposite to each other. This implies that the accuracy of SMGA locations is the order of SMGA size. Therefore, it may be difficult to distinguish the barrier based on the results from kinematic source in-
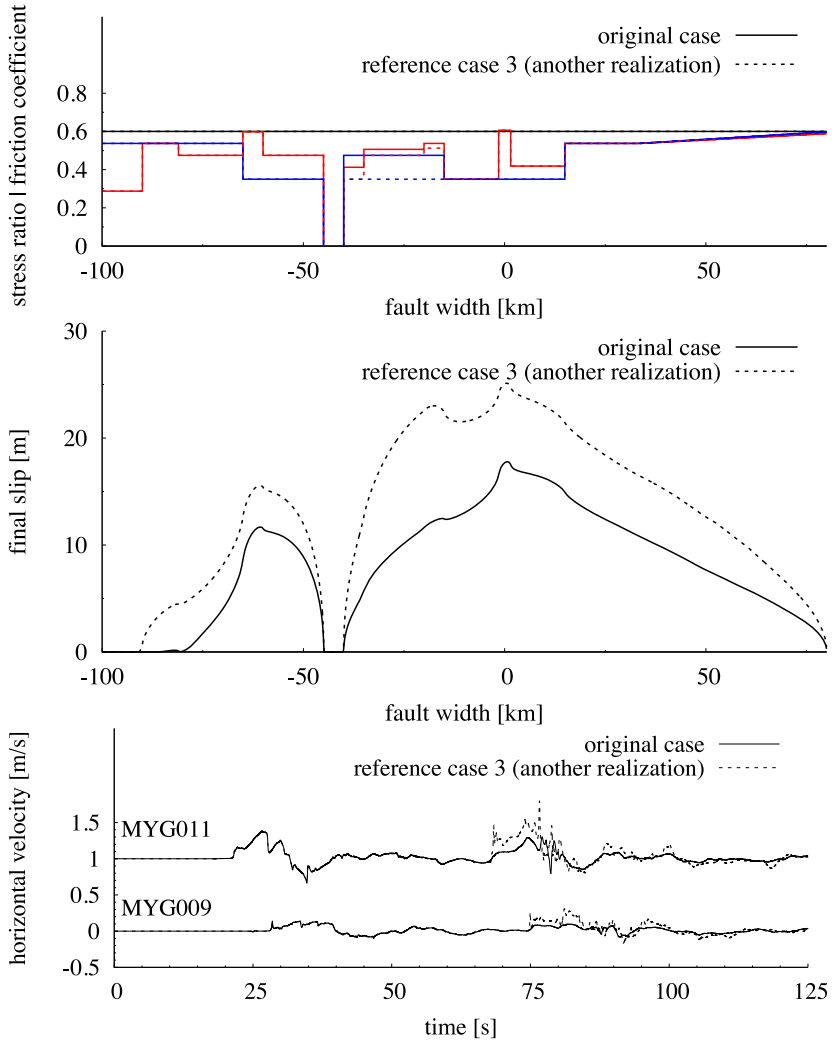

Fig. 12. Comparison of simulation results between the original case and the reference case 3 (another realization); friction coefficient (top), final slip distribution (middle) and synthetic velocity waveforms (bottom).

versions.

In order to clarify the effect of the free surface on the generation of the second significant event, we perform two other reference simulations, case 1: the same dynamic parameters without a free surface, and case 2: different dynamic parameters with a free surface. The velocity model beneath a depth of $0 \mathrm{~km}$ is set to be the same model as in the previous simulation, and the model above $0 \mathrm{~km}$ for case 1 is equal to the velocities of the uppermost layer of the previous model. The dynamic parameters, fault geometry, and calculation conditions are the same as in the previous simulation for case 1 , while both $\mu_{0}$ and $\mu_{r}$ from -40 to $-20 \mathrm{~km}$ are reduced to 0.35 for case 2 .

Figure 10 shows the final slip distribution and velocity waveforms for case 1 compared to the previous results, denoted as the "original case". Notice that the displayed synthetic waveforms for case 1 are multiplied by a factor of two to account for twice the amplification on the free surface. Final slips for the first significant event at around $-60 \mathrm{~km}$ are similar to each other, whereas slips for the second event are insignificant. Likewise, for the second wave group, the final slip distribution is only significant in the model with a free surface. Therefore, the effect of the free surface is essential to the generation of the second events.

Figure 11 shows the dynamic parameters, final slip distributions, and velocity waveforms, for case 2 . The effect on the free surface is considered in this case, whereas the second wave group is not significant. When the second signifi- 
cant event is triggered in the original simulation, the rupture front has just arrived at the shallower fault edge. If the origin of the second wave group is the arrival of the rupture at the free surface, the second wave group would appear in the result for case 2 .

The accuracy of the simulated result in the shallower region may not be well guaranteed. Furthermore, the modeled fault does not reach the free surface. These factors result in an underestimation of the final slips in the shallower region compared to the inversion results. However, we point out that a significant effect from the shallower region is not required for the multi-event generation.

Notice that the dynamic parameters are not validated by comparing the amplitudes between observed and synthetic waveforms because the 2D $P-S V$ wave field does not simulate the actual geometric spreading in 3D. The parameter set of our original model is the best we have found, but this may not be the optimum set. Therefore, it is worth showing the justifiability of our choice of parameter set.

In case $3, \mu_{p}, \mu_{r}$, and $D_{C}$ from $-40 \mathrm{~km}$ to $-20 \mathrm{~km}$ are set to be $0.6,0.35$ and $0.8 \mathrm{~m}$, respectively, which are the same as the values from -65 to $-45 \mathrm{~km}$. The model gives the same fracture energy with the original model at the area corresponding to the generation of the second significant event. The initial stress ratio is modified to control the rupture time at the area. Figure 12 shows the dynamic parameters, final slip distributions and velocity waveforms. The area from $-40 \mathrm{~km}$ to $-20 \mathrm{~km}$ is ruptured, and it generates the second significant event. As seen in the comparison of the waveforms, the second wave group is clearly observed at the same time as the result for the original case. The amplitudes of the second wave group are about twice larger than in the original case, because a larger stress drop is assumed. This implies that the absolute values of dynamic parameters for the second significant event cannot be determined quantitatively, and 3D simulations of the dynamic rupture and the seismic wave propagation are required. However, we emphasize that the generation mechanism of the second significant event is not directly related to the values because of the causality.

\section{Conclusion}

We simulate the dynamic rupture process that occurred during the 2011 off the Pacific coast of Tohoku Earthquake on the cross-section passing through the hypocenter. The first significant event occurred at around $-60 \mathrm{~km}$ along the fault width direction. The waves, scattered mainly from the free surface, come back to the fault plane in about $25 \mathrm{~s}$, and generate a stick-slip with small amplitude around the hypocenter. The stick-slips expand the ruptured area to the deeper side, and then the second significant event is triggered. The synthetic waveforms consist of two major wave groups, which are similar to the observations.

Acknowledgments. We thank two anonymous reviewers and the editor Prof. Takashi Furumura for their thorough comments. We are grateful to the following persons for the useful information they provided us and for helpful discussions: Professor Kazuro Hirahara, Professor Sumio Sawada, Professor Shin'ichi Miyazaki, Dr. Kimiyuki Asano, Dr. Wataru Suzuki, Dr. Hiroe Miyake, and many other researchers. This study was supported in part by the Grant-in-Aid for Young Scientists B of the Japan Society for the Promotion of Science. We offer our thanks to NIED for the contributions to the strong ground motion observations, namely, K-NET, KiK-net.

\section{References}

Aochi, H. and E. Fukuyama, Three-dimensional nonplanar simulation of the 1992 Landers earthquake, J. Geophys. Res., 107, 2039, 2002.

Aoi, S., T. Kunugi, W. Suzuki, N. Morikawa, H. Nakamura, N. Pulido, K. Shiomi, and H. Fujiwara, Strong motion characteristics of the 2011 Tohoku-oki earthquake from K-NET and KiK-net, Proc. of 2011 SSA Annual Meeting, 2011.

Asano, K. and T. Iwata, Source model for strong ground motion generation in the frequency range $0.1-10 \mathrm{~Hz}$ during the 2011 Tohoku earthquake, Earth Planets Space, 64, this issue, 1111-1123, 2012.

Dalguer, L. A. and S. M. Day, Staggered-grid split-node method for spontaneous rupture simulation, J. Geophys. Res., 112, B02302, 2007.

Day, S. M., L. A. Dalguer, N. Lapusta, and Y. Liu, Comparison of finite difference and boundary integral solutions to three-dimensional spontaneous rupture, J. Geophys. Res., 110, B12307, 2005.

Duan, B., Dynamic rupture of the 2011 Mw9.0 Tohoku-oki earthquake: roles of a possible subducting seamount, J. Geophys. Res., 2012 (in printing).

Fukuyama, E. and R. Madariaga, Rupture dynamics of a planar fault in a 3D elastic medium: rate- and slip-weakening friction, Bull. Seismol. Soc. Am., 88, 1-17, 1998.

Fukuyama, E., R. Ando, C. Hashimoto, S. Aoi, and M. Matsu'ura, A physics-based simulation of the 2003 Tokachi-oki, Japan, earthquake to predict strong ground motions, Bull. Seismol. Soc. Am., 99, 3150-3171, 2009.

Goto, H., L. Ramírez-Guzmán, and J. Bielak, Simulation of spontaneous rupture based on a combined boundary integral equation method and finite element method approach: SH and P-SV cases, Geophys. J. Int., 183, 975-1004, 2010.

Graves, R. W., Simulating seismic wave propagation in 3D elastic media using staggered-grid finite differences, Bull. Seismol. Soc. Am., 86, 1091-1106, 1996

Harris, R. A., M. Barall, R. Archuleta, E. Dunham, B. Aagaard, J. P. Ampuero, H. Bhat, V. Cruz-Atienza, L. Dalguer, P. Dawson, S. Day, B. Duan, G. Ely, Y. Kaneko, Y. Kase, N. Lapusta, Y. Liu, S. Ma, D. Oglesby, K. Olsen, A. Pitarka, S. Song, and E. Templeton, The SCEC/USGS dynamic earthquake rupture code verification exercise, Seismol. Res. Lett., 80, 119-126, 2009.

Ida, Y., Cohesive force across the tip of a longitudinal-shear crack and Griffith's specific surface energy, J. Geophys. Res., 77, 3796-3805, 1972.

Ide, S. and M. Takeo, Determination of constitutive relations of fault slip based on seismic wave analysis, J. Geophys. Res., 102, 27379-27391, 1997

Ide, S., A. Baltay, and G. C. Beroza, Shallow dynamic overshoot and energetic deep rupture in the $2011 \mathrm{Mw} 9.0$ Tohoku-Oki earthquake, Science, 332, 1426-1429, 2011.

Ishii, M., High-frequency rupture properties of the $M_{\mathrm{w}} 9.0$ off the Pacific coast of Tohoku Earthquake, Earth Planets Space, 63, 609-614, 2011.

Ito, A., G. Fujie, S. Miura, S. Kodaira, R. Hino, and Y. Kaneda, Bending of the subducting oceanic plate and its implification for rupture propagation of large interplate earthquake off Miyagi, Japan, in the Japan trench subduction zone, Geophys. Res. Lett., 32, L05310, 2005.

Kaneko, Y., J. P. Ampuero, and N. Lapusta, Spectral-element simulations of long-term fault slip: effect of low-rigidity layers on earthquake-cycle dynamics, J. Geophys. Res., 116, B10313, 2011.

Kase, Y. and S. M. Day, Spontaneous rupture processes on a bending fault, Geophys. Res. Lett., 33, L10302, 2006.

Kikuchi, M. and H. Kanamori, Inversion of complex body waves, Bull. Seismol. Soc. Am., 72, 491-506, 1982.

Koketsu, K., Y. Yokota, N. Nishimura, Y. Yagi, S. Miyazaki, K. Satake, Y. Fujii, H. Miyake, S. Sakai, Y. Yamanaka, and T. Okada, A unified source model for the 2011 Tohoku earthquake, Earth Planet. Sci. Lett., 310, 480-487, 2011.

Koper, K. D., A. R. Hutko, T. Lay, C. J. Ammon, and H. Kanamori, Frequency-dependent rupture process of the $2011 M_{\mathrm{W}} 9.0$ Tohoku Earthquake: Comparison of short-period $P$ wave backprojection images and broadband seismic rupture models, Earth Planets Space, 63, 599_ 602, 2011.

Kurahashi, S. and K. Irikura, Source model for generating strong ground 
motions during the 2011 off the Pacific coast of Tohoku Earthquake, Earth Planets Space, 63, 571-576, 2011.

Levander, A. R., Fourth-order finite-difference P-SV seismograms, Geophysics, 53, 1425-1436, 1988.

Ludwig, W. J., J. E. Nafe, and C. L. Drake, Seismic refraction, in The Sea, vol. 4, 54-84, Wiley-Inter-science, 1970.

Miura, S., N. Takahashi, A. Nakanishi, T. Tsuru, S. Kodaira, and Y. Kaneda, Structural characteristics off Miyagi forearc region, the Japan Trench seismogenic zone, deduced from a wide-angle reflection and refraction study, Tectonophysics, 407, 165-188, 2005.

Miyazaki, S., J. J. McGuire, and P. Segall, Seismic and aseismic fault slip before and during the 2011 off the Pacific coast of Tohoku Earthquake, Earth Planets Space, 63, 637-642, 2011.

Nakahara, H., H. Sato, T. Nishimura, and H. Fujiwara, Direct observation of rupture propagation during the 2011 off the Pacific coast of Tohoku Earthquake $\left(M_{\mathrm{w}} 9.0\right)$ using a small seismic array, Earth Planets Space, 63, 589-594, 2011.

Oglesby, D. and R. Archuleta, Dynamics of dip-slip faulting: explorations in two dimensions, Geophys. J. Int., 105, 13643-13653, 2000.

Page, M., P. M. Mai, and D. Schorlemmer, Testing earthquake source inversion methodologies, Eos Trans. AGU, 92, 75, 2011.

Sato, M., T. Ishikawa, N. Ujihara, S. Yoshida, M. Fujita, M. Mochizuki, and A. Asada, Displacement above the hypocenter of the 2011 TohokuOki earthquake, Science, 332, 1395, 2011.

Sawada, S., C. S. Fu, T. Kitano, and S. Yoshikawa, Multi-event inversion analysis for simpler representation of source mechanism, Proc. of 10th WCEE, 757-760, 1992.

Sekiguchi, H., K. Irikura and T. Iwata, Fault geometry at the rupture termination of the 1995 Hyogo-ken Nanbu earthquake, Bull. Seismol. Soc. Am., 90, 117-133, 2000.
Suzuki, W., S. Aoi, H. Sekiguchi, and T. Kunugi, Rupture process of the 2011 Tohoku-Oki mega-thrust earthquake (M9.0) inverted from strongmotion data, Geophys. Res. Lett., 38, L00G16, 2011.

Tada, T. and T. Yamashita, Non-hypersingular boundary integral equations for two-dimensional non-planar crack analysis, Geophys. J. Int., 130, 269-282, 1997.

Wada, K. and H. Goto, Generation mechanism of surface and buried faults: effect of plasticity in a shallow crust structure, Bull. Seismol. Soc. Am., 2012 (in printing).

Wang, D. and J. Mori, Rupture process of the 2011 off the Pacific coast of Tohoku Earthquake $\left(M_{\mathrm{w}} 9.0\right)$ as imaged with back-projection of teleseismic $P$-waves, Earth Planets Space, 63, 603-607, 2011.

Yamamoto, Y., R. Hino, and M. Shinohara, Mantle wedge structure in the Miyagi Prefecture forearc region, central northeastern Japan arc, and its relation to corner-flow pattern and interplate coupling, J. Geophys. Res., 116, B10310, 2011.

Yoshida, K., K. Miyakoshi, and K. Irikura, Source process of the 2011 off the Pacific coast of Tohoku Earthquake inferred from waveform inversion with long-period strong-motion records, Earth Planets Space, 63, 577-582, 2011.

Yoshida, N., Earthquake Response Analysis of Ground, Kajima, Tokyo, 2010 (in Japanese).

Zhang, H., Z. Ge, and L. Ding, Three sub-events composing the 2011 off the Pacific coast of Tohoku Earthquake $\left(M_{\mathrm{W}} 9.0\right)$ inferred from rupture imaging by back-projecting teleseismic $P$-waves, Earth Planets Space, 63, 595-598, 2011.

H. Goto (e-mail: goto@catfish.dpri.kyoto-u.ac.jp), Y. Yamamoto, and S. Kita 